\title{
REDES SOCIAIS: UM NOVO MUNDO PARA OS IDOSOS
}

\author{
Camila Wasserman \\ Universidade Federal do Rio Grande do Sul. Faculdade de Educação. \\ camiwass@gmail.com \\ Tássia Priscila Fagundes Grande \\ Universidade Federal do Rio Grande do Sul. Faculdade de Educação. \\ tpri.fagundes@hotmail.com \\ Leticia Rocha Machado \\ Universidade Federal do Rio Grande do Sul. Programa de Pós-Graduação em \\ Informática na Educação. \\ leticiarmachado@yahoo.com.br \\ Patricia Alejandra Behar \\ Universidade Federal do Rio Grande do Sul. Programa de Pós-Graduação em \\ Informática na Educação. \\ pbehar@terra.com.br
}

\begin{abstract}
RESUMO: Ao longo dos anos ocorreram mudanças significativas na sociedade, principalmente em relação às tecnologias e a expectativa de vida. Os idosos estão cada vez mais utilizando as tecnologias, incluindo as redes sociais. Para este tipo de público, as redes sociais estão se tornando um local não apenas como forma de passatempo, mas como fonte de novos conhecimentos, comunicação e interações. O presente artigo relata uma pesquisa realizada sobre redes sociais com 27 idosos que participam de um curso de inclusão digital desenvolvido na Universidade Federal do Rio Grande do Sul (UFRGS). A pesquisa não tem o propósito de objetivar unicamente a melhoria das ferramentas nas redes sociais, mas sim de analisar questões relativas a aspectos psicológicos, gerontológicos e educacionais envolvidos.
\end{abstract}

Palavras-chaves: redes sociais, idosos, educação.

\section{NETWORKS: A NEW WORLD FOR THE ELDERLY}

ABSTRACT: Over the years significant changes have occurred in society, especially in relation to technology and life expectancy. The elderly are increasingly using technology, including social networks. For this type of public social networks are becoming a place not only as a hobby, but as a source of new knowledge, communication and interactions. This article reports a survey on social networks with 27 seniors participating in a course designed for digital inclusion at the Federal University of Rio Grande do Sul (UFRGS). The research is not intended to target only the improvement of social networking tools, but rather to analyze issues related to psychological, and educational gerontology involved.

Keywords: social networks, elderly, education. 


\section{INTRODUÇÃO}

No Brasil, como em todo o mundo, os idosos estão cada vez mais ocupando o seu espaço dentro da sociedade. Este público passou a participar de forma mais ativa no meio social. Com isso, os idosos vêm se tornando mais participativos nas comunidades: estudando, trabalhando, comprando, viajando e se incluindo com mais frequência no mundo virtual. Portanto, o número de pessoas mais velhas aprendendo a usar o computador vem aumentado constantemente.

No mesmo contexto, houve um aumento significativo no número de idosos que estão utilizando as redes sociais. O aprofundamento sobre o perfil desta parcela da população que está imersa na Internet se torna necessário em futuras pesquisas nas áreas envolvidas.

A quantidade de usuários ativos nas redes sociais muda constantemente, pois as mesmas sofrem mudanças permanentemente. Uma pesquisa realizada pelo $M B I$ Mayer\&Bunge Informática sobre redes sociais, no ano de 2011, destacou que no Brasil, as cinco redes sociais mais utilizadas são: Facebook, LinkedIn, Twitter, YouTube e Orkut. Dentro estes, o Facebook teve um aumento significativo de usuários a partir do ano de 2010. Já o Orkut, por sua vez, teve uma queda de mais de 7\% dos usuários em relação ao ano anterior (Administradores, 2012).

As redes sociais, primordialmente o Facebook, nunca foram pensadas especificamente para pessoas mais velhas, foram desenvolvidas para um público jovem. Com a expansão das redes sociais, crianças com 10 anos já possuem mais de um perfil nas mesmas, além dos pré-adolescentes. Mas, a faixa de usuários que ainda predomina nas redes é a dos 30 anos (Dias, 2010). No entanto, o número de usuários com mais de 65 anos vem crescendo percentualmente desde o ano de 2008. Hoje, os idosos representam mais de $3 \%$ da população de usuários das redes sociais brasileiras.

Com o crescimento significativo dos idosos na utilização das redes sociais, surgem então algumas indagações sobre o uso das mesmas. Desta forma é importante compreender as redes sociais como um espaço que possa proporcionar ao idoso liberdade suficiente para interagir e usufruir das possibilidades oferecidas. Alguns estudos sobre o tema já foram realizados, no entanto ainda há uma carência em relação aos aspectos educacionais (Machado 2008).

Sendo assim, o presente artigo pretende: definir o perfil de um grupo de idosos que utilizam as redes sociais na UFRGS; motivações que levaram estes a entrar nas redes sociais; possíveis dificuldades na utilização e a importância dos recursos na vida destes indivíduos.

Portanto, a seguir são delineados os principais pontos a ser abordados neste artigo. Desta forma, será tratado inicialmente aspectos que envolvem os idosos e o mundo tecnológico, seguido da metodologia, análise dos dados com a discussão dos mesmos e, finalizando com as considerações finais.

\section{O MUNDO TECNOLÓGICO E OS IDOSOS: POSSIBILIDADES DE INCLUSÃO}

A população brasileira vem aumentando gradativamente ao longo dos anos, nos últimos 50 anos, por exemplo, o número de brasileiros quase triplicou. Mas, de 
acordo com o IBGE (2010) o que sobressaiu foi o aumento de idosos no Brasil, passando de $4,7 \%$ da população a mais de $10 \%$ atualmente. Todo este processo vem gerando alterações, não somente na vida dos indivíduos, mas também da população em geral.

O que se percebe, é que o idoso está com desejo de convivência, abertura a grandes descobertas e produtividade. Esta população está disposta a ajudar os outros e estar de bem com a vida. Isso pressupõe desafiar o medo e vencer os desafios (Nunes, 2010, p.111)

Em paralelo, o uso das tecnologias aumentou na sociedade, em diferentes ambientes e faixas etárias. A busca e uso de tecnologias mais atualizadas como smartphone, reprodutores de mídias, jogos interativos, tablets, entre outros, evidenciouse nos últimos anos na população. Entre as tecnologias, os computadores também estão nesta lista, devido, principalmente, as variedades de recursos e interações que é proporcionado aos usuários. Os idosos também se incluem nesta busca pelo uso das tecnologias, onde a comunicação se destaca como principal objetivo. Ou seja, o computador

[...] ao longo dos anos, tem comprovado que é uma ferramenta de diversas possibilidades de formas de atuação e atualização. Tem também se mostrado, como auxilio a novos modelos de organização da vida, com a otimização do tempo de dispêndio para realização de trabalhos e tarefas (Kachar, 2003 apud Behar, Machado, Ribeiro, Ebeling, 2010, p.112).

Neste sentido, a informática é mais do que apenas um passatempo para os idosos,

A informática, ao longo dos anos, tem comprovado que é uma ferramenta de diversas possibilidades de formas de atuação e atualização. Tem também se mostrado, como auxilio a novos modelos de organização da vida, com a otimização do tempo de dispêndio para realização de trabalhos e tarefas (Kachar, 2003 apud Behar, Machado, Ribeiro, Ebeling, 2010, p.112).

Com o interesse dos idosos em aprender a utilizar os computadores, surge a necessidade de capacitar os professores a fim de buscar subsídios para atender esse novo publico alvo, suas características e exigências.

Segundo Nunes (2010), ao falar em informática para os idosos é extremamente importante a atuação de um professor qualificado, onde deve-se incluir o aluno neste novo universo digital com metodologias e abordagens adequadas para suas necessidades. O professor carece ensinar aos idosos assuntos de seu interesse, portanto é importante observar o aluno como um ser pensante, capaz de produzir e de tornar-se cada vez mais um cidadão ativo na sociedade globalizada.

Os idosos têm tornado o computador um grande aliado para estimular a memoria e construir/aprimorar os conhecimentos. Há alguns anos atrás, as pessoas mais velhas pouco utilizavam as tecnologias e, quando o faziam, era para se informar com as noticias, por exemplo. No entanto, atualmente, as redes sociais estão cada vez mais fazendo parte do cotidiano dos idosos.

As redes sociais são espaços destinados para a socialização, utilizando para isso recursos de compartilhamento de informações, fotos, vídeos e principalmente comunicação entre seus usuários. Nestes espaços é possível se criar também 
comunidades virtuais destinadas a assuntos específicos, como por exemplo: comunidade de animais domésticos, grupos de discussões de entretenimento entre outros. Portanto a colaboração se torna a palavra fundamental quando se fala sobre redes sociais.

Estes tipos de redes são estudadas desde 1930 na literatura pertinente, passando por diferentes aportes teóricos que tratam sobre o tema. Uma rede social é formada em diferentes culturas e sociedades, geralmente constituída por comunidades como: a família, escola, emprego etc. A partir do surgimento da internet na década de 90 , foi possível a expansão das redes sociais através do uso das tecnologias comunicacionais como e-mail, messengers. Com o aprimoramento da internet, ou seja, a segunda geração (web 2.0) ocorreu mudanças significativas nas formas de comunicação e interação. A partir destas transformações foi desenvolvido o Orkut (em 2004), inovando no setor das redes sociais através das possibilidades de compartilhamento de informações e comunicação, além de criar laços entre os "amigos virtuais" (Aguiar, 2006; Couto, Rocha, 2010).

Portanto, a partir destas mudanças, novos tipos de redes estão surgindo, trazendo mais recursos que atraíram os seus usuários, entre eles os idosos. Nos Estados Unidos já existem redes sociais voltadas principalmente para pessoas com mais de 50 anos. É oferecido um espaço planejado para essa faixa etária, propiciando jogos para estimular o potencial cerebral, contendo dicas sobre a saúde do idoso e alimentação adequada, fóruns de discussões e outros temas de interesse deste público (Dias, 2010).

Um dos principais motivos dos idosos utilizarem as novas tecnologias é a possibilidade de estarem incluídos na sociedade, ou seja, ser ativo e fazer parte do novo panorama. Um dos meios para inserir-se neste mundo virtual é por meio das redes sociais. Com as mudanças paradigmáticas, as pessoas idosas vêm conquistando o seu espaço neste mundo virtual. As redes sociais para este público estão se tornando um local não apenas como forma de passatempo, mas como fonte de novos conhecimentos e, em muitos casos, divulgação de seus trabalhos voluntários, direitos dos idosos, potencialidades artísticas e intelectuais.

A partir da inserção nas redes sociais, os idosos passam a querer descobrir diferentes formas de comunicação. "A descoberta de poder se comunicar com amigos e parentes em qualquer lugar do mundo desperta um grande fascínio em cada um. Com isso, surge a vontade de conhecer mais e realizar novas conexões" (Dias, 2010).

É possível observar o quanto o idoso vem conquistando o seu espaço no mundo digital. As empresas, aos poucos, estão se conscientizando que existem muitas possibilidades para esta faixa etária e, cada vez mais, repensando como melhorar seus produtos e espaços para atender as necessidades dos idosos.

Neste panorama repensar as práticas pedagógicas para o uso das redes sociais com idosos é fundamental. Maissiat e Biazus (2000) afirmam que é preciso construir estratégias metodológicas educacionais para preparar a população idosa para a utilização e domínio dos recursos tecnológicos. Portanto, deve-se, prioritariamente, procurar quais abordagens são adequadas para introduzir o idoso neste novo universo.

Este artigo pretende apresentar formas de utilização das redes sociais pelos idosos. Objetivando desta forma, desenvolver competências que abranjam além da simples técnica no uso das ferramentas tecnológicas, mas que possibilitem o seu uso de forma crítica e reflexiva. A seguir será apresentada a metodologia adotada neste artigo.

\section{METODOLOGIA}


Muitas indagações surgiram ao pesquisar aporte teórico sobre o uso de redes sociais pelos idosos. Dentre as questões, a motivação que leva o idoso a utilizar a rede social é um aspecto relevante e de suma importância. E a resposta a esta indagação possibilitará entender as necessidades e formas de utilização educacional das redes pelos os idosos.

Desta forma, o presente artigo foi desenvolvido em uma abordagem qualitativa quantitativa como forma de realizar uma pesquisa ampla do tema. A pesquisa foi realizada com um grupo de 27 idosos com média de idade igual ou mais de 67 anos, sendo apenas 3 do gênero masculino e o restante do gênero feminino. Este grupo participa de um curso de inclusão digital desenvolvido na Universidade Federal do Rio Grande do Sul. Este é desenvolvido de forma presencial, sendo composto por grupos de, no máximo, 10 pessoas. Cada aula possui duração de $2 \mathrm{~h}$, onde é realizado, inicialmente, um levantamento dos temas que os idosos desejam aprender ou aprofundar. O curso é oferecido desde 2009 de forma continuada.

A coleta de dados ocorreu a partir de questionários e observação presencial e virtual. O questionário aplicado possuía 8 perguntas, sendo elas de múltipla escolha e dissertativas. O registro da coleta de dados virtuais foi realizado na rede social (Facebook) no qual os grupos de idosos estão inseridos e que também se encontram as autoras deste artigo.

Os dados de natureza qualitativos foram tratados a partir da análise de conteúdo, conforme Bardin (2004). Já os dados de natureza quantitativos foram analisados a partir da distribuição de frequência representada em percentuais, média e desvio padrão. Essas duas formas de análise foram escolhidas, para que houvesse uma complementação nos dados coletados, no que tange os objetivos propostos.

A partir da coleta e análise dos dados foi possível delinear o perfil do grupo participante em redes sociais. A seguir serão descritos os resultados obtidos nesta pesquisa e suas contribuições para áreas como gerontologia, educação e informática.

\section{DISCUSSÃO E ANÁLISE DE DADOS}

Com o aumento do uso das redes sociais pelos idosos, os dados coletados para este estudo possibilitou iniciar um discussão mais aprofundada sobre o tema. Portanto é possível analisar e observar os diversos motivos, dificuldades e funcionalidades que mais aparecem na utilização das redes sociais pela população mais velha. A partir dos dados coletados, foi possível criar algumas categorias de análise, conforme será abordado a seguir.

Em relação às redes sociais pode-se observar que, a partir dos dados coletados, há uma predominância no uso do Facebook, o que totalizou 75\% (Figura 1) 


\section{Redes sociais utilizadas}



Figura 1: Percentual das redes sociais utilizadas

Fonte: As autoras (2012)

O fato do Facebook se destacar como uma das redes sociais mais utilizadas pelos idosos se deve por diferentes fatores. Um dos aspectos é o fato de adotar recursos com títulos e conteúdos na língua portuguesa (por exemplo, jogos, botões de uso de adição entre outros). Outro fator a simplicidade de postar e se comunicar com facilidade com os amigos e familiares, incluindo o popular "Curtir" nos conteúdos e comentários, sendo uma maneira prática de manifestação dos usuários. Conforme cita um idoso "Facebook ,porque acho que é menos complicado para usá-lo".

No ano de 2010 foi lançado o filme ganhador de três prêmios Oscar "The Social Network" (conhecido no Brasil como "A Rede Social"). Neste filme é abordado a fundação do Facebook, o que elevou o índice de cadastros que já vinha aumentando, fazendo com que mais pessoas aderissem (Landim, 2011).

Estas razões também contribuíram para a popularidade desta rede social entre os idosos. No entanto, apesar do Facebook ser ainda a mais utilizada, o Twitter também é citado como uma rede social conhecida (Figura 1).

Nas respostas e participação na rede social, os idosos mostraram uma preocupação com a questão da privacidade. O e-mail e messengers são citadas como ferramentas preferenciais por tratarem, de acordo com os participantes, de serem menos invasivos na vida deles.

A privacidade ainda é um tema que gera muitos debates nas redes sociais. A facilidade de desenvolver "amizades" intimida os idosos, uma vez que esta parcela da população está adaptada a construir amizades baseada em relações presenciais a partir de diálogos, confissões e confiança, diferentemente de muitas relações desenvolvidas nas redes sociais. Conforme diz Sonia (2007, p.11) "[...] a palavra "amigos" foi ganhando um sentido muito diferente das relações afetivas tradicionais, que pressupõem reciprocidade, confiança, intimidade, sinceridade, e sendo associada a uma competitividade antagônica ao espírito solidário das redes sociais da vida cotidiana e dos contextos sociopolíticos".

Já em relação à frequência no uso das redes sociais, observa-se que os idosos estão mais ativos na sua utilização, pois responderam, na sua maioria, que entram mais 
de duas vezes por semana (64\%). Este dado mostra a importância de se aprofundar a discussão sobre o tema (Figura 2).

\section{Frequência no uso das Redes sociais}

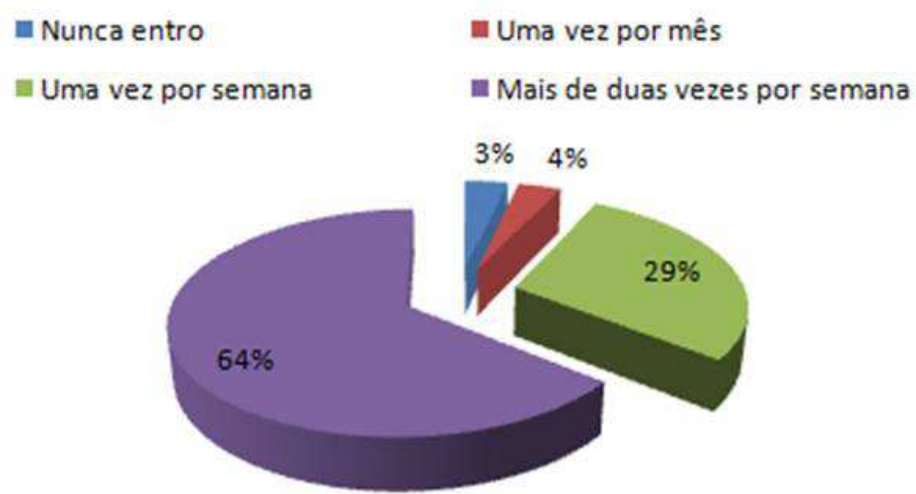

Figura 2: Percentual do uso das redes sociais Fonte: As autoras (2012)

Em relação às ferramentas disponíveis nas redes sociais, predominou o uso de mensagens instantâneas (30\%), seguido de fotos (28\%) e publicações no mural (16\%) (Figura 3). Isso mostra que a comunicação ainda é o foco dos idosos.

\section{Ferramentas utilizadas nas Redes sociais}

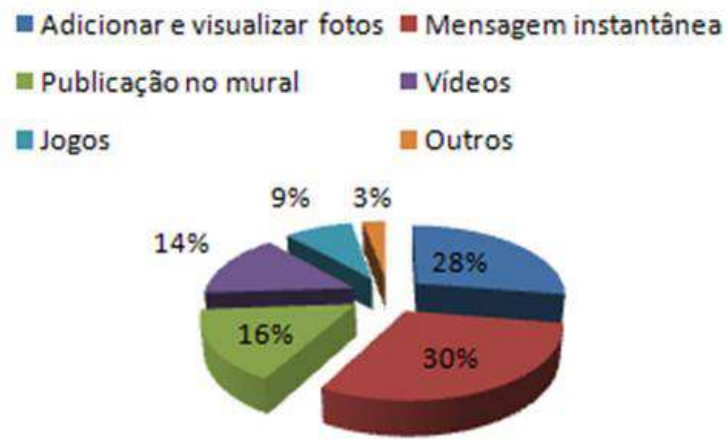

Figura 3: Percentual das ferramentas utilizadas nas redes sociais Fonte: As autoras (2012)

A comunicação é primordial para idosos, uma vez que permite a socialização e participação, principalmente com familiares que em muitos casos se distanciam dos seus pais/avós. As tecnologias de informação e comunicação (TIC) possibilitaram esta comunicação mais rápida com os amigos e familiares. Com fenômeno das redes sociais, a comunicação se intensificou e aproximou mais as pessoas.

Na revista Unati (2002) é afirmado que a comunicação possui uma importância para os idosos. Portanto é um dos meios essenciais para entender e lidar com outros indivíduos, minimizando carências emocionais e afetivas nas interações. 
Estes dados são corroborados nas outras respostas realizadas pelo grupo. Em relação às mudanças que as redes sociais provocaram nas suas rotinas, apenas 5 relataram que não houve mudança. Uma parte do grupo citou que as redes sociais acabaram exigindo mais tempo de dedicação e foi apontado como um ponto negativo no uso destes. O restante do grupo apontou que as redes sociais influenciaram na comunicação (12) e visualização das atualizações de amigos e familiares (8). Como citou uma pessoa idosa "E gostoso assistir os vídeos que interessa, ver as amigas $e$ parentes mandando recados".

Sobre as dificuldades apontadas, $22 \%$ dos idosos responderam que não possuem nenhuma no uso das ferramentas e $78 \%$ possuem dificuldade no manuseio. Conforme cita um idoso sobre o uso das mesmas e suas dificuldades "Não, ainda não lido com tudo que já tem... Alias seria bom que tivesse um professor a nossa disposição, como no google, nós faríamos as perguntas e eles nos dava as respostas passo a passo... joia né?".

Dentre as dificuldades apontadas estão a inserção de vídeos e fotos nas redes sociais e o receio da exposição excessiva. Este receio com a exposição é possível de se notar nas postagens dos idosos nas redes, onde os mesmos acabam utilizando ferramentas que restringem a comunicação apenas com pessoas específicas. Como expos um idoso "O temor de que o uso da rede social leve a uma exposição pessoal indesejada pode ser um fator adicional para minha baixa utilização". Um fator que influencia o medo à exposição é o fato de que a maioria dos idosos recebe e-mails salientando que as redes sociais, em específico o Facebook, proporcionava a invasão de hackers nos dados de identificação gerando um princípio de pânico entre o grupo. Após uma explicação do professor, houve uma diminuição no receio de uso das redes sociais, mas que ainda acompanha muitos no seu uso.

Portanto, "O motivo dessa mudança na rotina dos idosos está relacionado não somente à necessidade que eles têm de se inserir socialmente, mas também por auxiliar na interface de uma aproximação com seus familiares" (Nunes, 2010, p.120). Completando com o que um idoso citou sobre a mudança que as redes realizaram na sua rotina: "Com toda a certeza, assim posso ver fotos dos meus filhos que moram longe, posso falar com eles e mandar mensagens. Enfim ficar em contato com meus filhos, irmãos e amigos". Ou mesmo como expos outro idoso "Encontrei amigos que mto, mto tempo não tinha noticia nenhuma. É muito gratificante..."

\section{CONSIDERAÇÕES NÃO FINAIS}

Ao longo dos anos a sociedade se preocupou em desenvolver tecnologias que facilitassem o cotidiano do ser humano. Desde o surgimento da Internet mais recursos foram construídos e acabaram fazendo parte da vida das pessoas, se tornando, em muitos casos indispensáveis para atividades rotineiras. Apesar desta evolução, a sociedade não se preocupou em acompanhar a expectativa crescente na idade da população, o que formou um grupo de excluídos da sociedade informatizada.

Culturalmente ainda não há uma preocupação com a inclusão digital de pessoas mais velhas, o que não impediu os idosos em procurarem cursos que possibilitassem uma capacitação no uso das ferramentas, incluindo as redes sociais. 
Apesar da presente pesquisa se restringir a um grupo de 27 participantes, foi possível fazer um primeiro mapeamento de dados sobre este tipo de público e sua relação com as redes sociais, suas dificuldades e motivações para o uso.

Portanto, a partir dos dados coletados, observa-se que a rede social mais usada é o Facebook, por ser intuitivo e se tratar de um espaço de fácil utilização para os idosos.

Entre as ferramentas citadas e as mais utilizadas estão as de mensagens instantâneas, seguidos do compartilhamento de fotos e mensagens. $\mathrm{O}$ que significa uma preocupação em estar mais perto de amigos e familiares, além do compartilhamento de informações com estes. Portanto, pode-se perceber que os idosos ainda procuram as tecnologias como forma de comunicação com os familiares e amigos, o que significa uma preocupação com o isolamento e desatualização dos acontecimentos que os dizem respeito.

O que se destacou neste estudo foi a preocupação dos idosos com a sua privacidade nas redes sociais. Este medo se deve, em muitos casos, pelo receio da exposição excessiva de vida pessoal para os integrantes das redes. Estes dados são muito importantes, principalmente para educadores que trabalham na área, pois podem auxiliar no desenvolvimento das aulas sobre o tema de forma a esclarecer as possibilidades de segurança nas redes sociais. A partir disto evidencia o valor de desenvolver cartilhas explicativas específicas para os idosos e que esclarecessem mais o público.

Realizar estudos sobre este tipo de público e as tecnologias considera-se primordial. A cada ano surgem novas redes sociais e novos recursos. Ao mesmo tempo, os idosos estão cada vez mais presentes neste meio e suas angustias, motivações os acompanham. Portanto, as pesquisas não devem ser realizadas apenas objetivando a melhoria das ferramentas nas redes sociais, mas também sobre questões relativas aos aspectos psicológicos, gerontológicos e educacionais envolvidos.

Portanto, entende-se que e trata de uma investigação com considerações não finais e que abre um leque de possibilidades de trabalho em relação aos idosos e possibilidades educacionais.

\section{REFERÊNCIAS BIBLIOGRÁFICAS}

ADMINISTRADORES: o portal da administração. Estudo revela que usuários de redes sociais estão mais preocupados com a privacidade. Disponível em: http://www.administradores.com.br/informe-se/tecnologia/estudo-revela-que-usuariosde-redes-sociais-estao-mais-preocupados-com-a-privacidade/48002/. Acesso em: 05 maio 2012.

AGUIAR, Sonia. Redes sociais e tecnologias digitais de informação e comunicação: relatório de pesquisa. Rio de Janeiro: NUPEG, 2006.

AGUIAR, Sonia. Redes sociais na internet: desafios à pesquisa. XXX Congresso Brasileiro de Ciências da Comunicação. Santos: Intercom - Sociedade Brasileira de Estudos Interdisciplinares da Comunicação, 29 de agosto a 02 de setembro de 2007.

BAMBINA, Antonina. The interplay of social networks and computer mediated communication. Disponível em: http://www.worldcat.org/title/online-social-supportthe-interplay-of-social-networks-and-computer-mediated-

communication/oclc/443283769?referer=\&ht=edition. Acesso em: 10 de maio de 2012. 
BARDIN, Lawrence. Análise de Conteúdo. Lisboa: Portugal. 2004.

COUTO, Edvaldo Souza; ROCHA, Telma Brito (Orgs). A vida no Orkut: Narrativas e aprendizagens nas redes sociais. Salvador: EDUFBA, 2010.

DIAS, Fernanda. Idosos aderem as redes sociais e passam a produzir conteúdo para a web. Disponível em: http://opiniaoenoticia.com.br/vida/comportamento/idososaderem-as-redes-sociais-e-passam-a-produzir-conteudo-para-a-web/. Acesso em 10 de maio de 2010.

LANDIM, Wikerson. 2011. 10 razões que tornaram o Facebook a rede social mais popular do Brasil. Disponível em: http://www.tecmundo.com.br/facebook/13347-10razoes-que-tornaram-o-facebook-a-rede-social-mais-popular-do-brasil.htm. Acesso em 13 maio 2012.

MAISSIAT, J. ; BIAZUS, M. C. V. Comunicação entre Gerações: uso das novas tecnologias da informação e comunicação como meio de inclusão social de idosos. In: TERRA, Newton Luiz; FERREIRA, Anderson Jackle; TACQUES, Cláudia de Oliveira, MACHADO Letícia Rocha (Orgs.). Envelhecimento e suas Múltiplas áreas do Conhecimento. Porto Alegre: EDIPUCRS, 2010.p. 89-93.

MACHADO, V. V. O uso da internet por idosos: um estudo netnográfico em Redes de comunicação online. 2008. Graduação [Trabalho de Conclusão], Universidade do Vale do Itajaí/UNIVALI, 2008.

NUNES, Vivian Patricia Caberlon. Envelhecimento: olhando-se no espelho da vida, através da inclusão digital. In: TERRA, Newton Luiz; FERREIRA, Anderson Jackle; TACQUES, Cláudia de Oliveira, MACHADO Letícia Rocha (Orgs.). Envelhecimento e suas Múltiplas áreas do Conhecimento. Porto Alegre: EDIPUCRS, 2010. p.109117. 